\title{
A PESAR DE LAS ESTADÍsTICAS, EL NÚMERO DE NIÑOS MUERTOS EN COLOMBIA POR DESNUTRICIÓN ES MENOR A LO ESPERADO
}

\author{
JORGE ENRIQUE PÉREZ CÁRDENAS \\ Director \\ Revista Biosalud \\ Departamento de Ciencias Básicas \\ Facultad de Ciencias para la Salud \\ Universidad de Caldas
}

La desnutrición y la muerte infantil asociada con este problema en Colombia es la consecuencia de la pobreza extrema en primer término, pero también de una serie de factores que la agudizan y que, en mi criterio, se presentan con una frecuencia más baja de lo esperado, a pesar de la reacción mediática y de las estadísticas.

La pobreza extrema en nuestro país es un aspecto que ya se ha determinado por parte del Departamento Administrativo Nacional de Estadística en Colombia (DANE); estudios realizados en el año 2015 muestran como la pobreza extrema en todo Colombia es del 7,9\%; sin embargo, a pesar de este bajo valor, la misma es mucho mayor en zonas rurales, con un 18\%. La región de La Guajira presenta aspectos particulares con respecto al resto del país que hacen que sea posible una mayor desnutrición en esta región, la cual se ha calculado que es del $80 \%$ en niños. Lo más importante quizás sean las condiciones de supervivencia de las comunidades indígenas ya que viven en unas zonas inhóspitas sin agua; la falta de agua, elemento indispensable para la supervivencia de cualquier ser vivo, hace que estas comunidades sean nómadas y además no puedan subsistir de la agricultura; de igual manera, la tenencia de animales se ve restringida por la ausencia de este vital líquido.

En el estudio realizado por el DANE, relacionado con pobreza extrema y multidimensional en Colombia, se demuestra que la zona pacífica, exceptuando el Valle del Cauca y la zona Atlántica, son regiones donde hay mayor pobreza multidimensional; siendo mayor en la primera región (33,8\% vs 31,2\%). A pesar de la reacción mediática, se requiere que se haga un estudio que demuestre cual es la frecuencia de desnutrición en nuestro país por regiones, para priorizar de esta manera la ejecución de políticas que disminuyan la frecuencia de muertes asociadas con este fenómeno social.

A pesar de que estadísticas del DANE muestran que la cobertura de acueducto y alcantarillado en Colombia es del 98 y 77\% respectivamente, en zonas rurales el acueducto llega al 61\% de este grupo poblacional, mientras que el alcantarillado solamente al $26 \%$; este dato muestra una cobertura baja en ambos servicios y si además se le agrega el dato de que al $54 \%$ de la población le gusta tomar agua directamente de la llave y se tiene en cuenta que en gran parte de estos acueductos rurales no hay tratamiento adecuado del agua, este aspecto puede aumentar la frecuencia de infecciones intestinales, que no solo están asociadas con el consumo de agua, sino también con la inadecuada disposición de las aguas servidas que en estas zonas del país es crítica. El parasitismo y las infecciones intestinales son factores importantes en la producción de desnutrición, por lo tanto, la población en riesgo de presentar enfermedades infecciosas intestinales es alta, lo que agrega así un elemento adicional al estado nutricional de los niños.

En el estudio de calidad de vida del DANE del 2015, no se muestra cuál es la cobertura de acueducto y alcantarillado en La Guajira, pero asumo por lo antes mencionado que esta es menor al promedio de la región rural; de igual manera, el adecuado desecho de excretas también debe ser menor y, por tanto, la incidencia

DOI: $10.17151 /$ biosa.2016.15.1.1 
de infecciones intestinales y respiratorias que son tanto causa como consecuencia de la desnutrición, pueden ser la causa de la alta morbimortalidad en niños indígenas Wayú de dicha región.

$\mathrm{Al}$ analizar la frecuencia de muertes por grupo de edad, se observa que en menores de 5 años en 2015 se presentaron aproximadamente 8000 muertes, que corresponden al $4 \%$ del total de muertes en el año; la mayor incidencia se presentó en menores de 1 año, en los cuales ocurrieron 6700 muertes; es decir, el 84\% de las muertes en este grupo. Puede haber muchas causas de muerte asociadas con los niños, pero es previsible que gran parte de ellas estén relacionadas con la desnutrición, puesto que aumenta la probabilidad de muerte a medida que disminuye la edad.

Por lo tanto, teniendo en cuenta estos análisis estadísticos que presenta el DANE, se podría prever que las estadísticas de muerte en niños asociada a desnutrición sean más altas en Colombia; pero también estas estadísticas demuestran que este problema no es exclusivo de La Guajira, sino que en otras partes de Colombia también se debería estar presentando. Un buen punto de partida para determinar hacia donde deben estar enfocados los esfuerzos de salud pública y del Instituto de Bienestar Familiar en la disminución de la desnutrición infantil y en consecuencia de las muertes por esta causa, es el desglose por departamentos de estas muertes infantiles, aunque es obvio por lo dicho, que existen dos regiones altamente deprimidas que incluyen 10 departamentos de los 32 que tiene Colombia.

Es posible que las políticas públicas tendientes a disminuir la pobreza sean efectivas, ya que las estadísticas han mostrado que año tras año este índice ha disminuido; sin embargo, se requiere ser más incisivo en este aspecto. El suministro de alimentos a niños en hogares comunitarios y en escuelas puede estar dando buenos resultados, pero hay una desprotección en menores de un año de edad que son los que presentan un mayor número de muertes en niños menores de 5 años y que debe ser atacado mejorando la calidad de vida de estos niños desde diferentes frentes, producto de una investigación cuidadosa de los factores desencadenantes de este fenómeno. 